\title{
WEIGHTED AND LOGARITHMIC LEAST SQUARE METHODS FOR MUTUAL EVALUATION NETWORK SYSTEM INCLUDING AHP AND ANP
}

\author{
Kazutomo Nishizawa \\ Nihon University
}

\author{
Iwaro Takahashi \\ Tsukuba University
}

(Received May 15, 2007; Revised December 2, 2008)

\begin{abstract}
In this paper we show that the weighted least square (WLS) and the logarithmic least square (LLS) of various methods to solve the mutual evaluation network (MEN) system including the Analytic Hierarchy Process (AHP) and the Analytic Network Process (ANP), are superior to others. Both methods can solve MEN problems without any restrictions of the structure matrix of MEN. We also show that the WLS always gives positive solutions, which generalize the proof by Blankmeyer. We establish the error analysis of WLS and LLS. Especially, we give solving methods of MEN problems with different variances of errors by the WLS and the LLS.
\end{abstract}

Keywords: AHP, ANP, least square

\section{Introduction}

The evaluation systems such as the Analytic Hierarchy Process (AHP) and the Analytic Network Process (ANP) are unified to the mutual evaluation network (MEN) system. The structure of MEN is the following;

There is a set $N$ of objects $1,2, \cdots, n$. An object $i$ has its own weight $u_{i}(>0)(i=1, \cdots, n)$. They are to be evaluated mutually. Let $s_{i j}(>0)$ be the evaluation value of an object $i$ evaluated by an object $j$, which is represented by the formula

$$
u_{i} \fallingdotseq s_{i j} u_{j}
$$

Another representation of the above fact is

$$
u_{i} / u_{j} \fallingdotseq s_{i j}
$$

where $s_{i j}$ corresponds to the paired comparison value in the AHP [5].

We have the data $s_{i j}$ for $(j, i) \in E$, where $E$ is a set of ordered pairs of the objects belonging to $N$. The analysis of MEN is to estimate $u_{1}, \cdots, u_{n}$ by the given data $\left\{s_{i j} \mid(j, i) \in\right.$ $E\}$ based on (1.1) or (1.2).

The structure of MEN is well represented by a directed graph $G$ called the graph of MEN, whose node corresponds to an object and directed arc $(j, i)$ corresponds to an ordered pair $(j, i) \in E$ of objects. Here we assume that the graph $G$ is connected.

The MEN with $E=\{(i, j) \mid i, j=1, \cdots, n, i \neq j\}$ is considered to be the ordinary AHP (with complete information) [13]. But for the AHP there is the special restriction

$$
s_{j i}=1 / s_{i j} \quad \text { for }(i, j) \in E .
$$


The MEN graph of AHP (with complete information) is a complete directed graph. Of course, there is an AHP whose set of pairs of objects is a subset of the above $E$. Such an AHP is called the incomplete AHP [13].

As for the ANP there are many types of MEN graph. The $n \times n$ matrix $\boldsymbol{S}$, whose $(i, j)$ element is $s_{i j}$ for $(j, i) \in E$ and zeros otherwise, is the well-known supermatrix [7]. For the ANP there is the restriction

$$
\sum_{i=1}^{n} s_{i j}=1, \quad j=1, \cdots, n
$$

that is, the matrix $\boldsymbol{S}$ is a column stochastic.

Here we treat more general MEN methods without any restrictions such as (1.3) and (1.4). Let us call the matrix $S$ defined above as the matrix of MEN for the general case. The structure of MEN is determined by its graph $G$ or its matrix $\boldsymbol{S}$, and it is well known that the irreducibility of $\boldsymbol{S}$ is equivalent to the strong connectivity of $G$.

We note that we do not mean the hierarchy structure constructed from criteria and alternatives of AHP by the MEN graph of AHP. For example, the set of nodes in the MEN graph of AHP corresponds to the set of alternatives evaluated within a criterion.

We intend to show that as the analyzing method of MEN problems, the logarithmic least square (LLS) method (see §2) and the weighted least square (WLS) method (see §2) are superior to other methods. Both methods can be applied to any MEN problem almost without any conditions, while the eigenvector method (see $\S 2$ ) cannot be directly applied to a MEN problem whose graph is not strongly connected. Further, both methods always give positive solutions and have error analyzing methods. We cannot analyze errors by almost any other method.

The criticism that the LLS method is not sensitive to inconsistencies included in $\left\{s_{i j}\right\}$ is given in [6], but this is valid only for the case of AHP with complete information, and loses grounds for general MEN problems.

The WLS method was proposed in [2] and the positivity of solutions was proved in [1], but this proof is valid only for the case where the matrix $\boldsymbol{X}^{T} \boldsymbol{X}$ (see $§ 3$ ) is non-singular and its off-diagonal elements are all negative. In $\S 3$ we prove the positivity for general MEN problems. In $\S 4,(\S 5)$ the error analysis on the LLS (the WLS) method is described. We often encounter the case where errors have different weights (or variances). In $\S 6$ error analyses for such cases are stated and this is applied to a typical ANP called the mutual evaluation problem. We can solve an ANP with a reducible supermatrix, that is, whose graph $G$ is not strongly connected, by the general method [10,14], but this structure is rather complicated. But by the WLS method or the LLS method we can directly solve such a reducible ANP. This is shown in $\S 7$ with the error analysis.

\section{The Analyzing Methods of MEN}

Here we state five analyzing methods of MEN problems; the minimax (or the eigenvector) (MIN), the averaged least square (ALS), the LLS, the WLS and the minimum $\chi^{2}$ (chi square) (MCS) method.

(i) The MIN method is well known and the most popular method, which was first proposed by $T$. Saaty [5]. This gives the solution by the eigenvector method, and is proved to be based on the mini-max principle by Frobenius $[8,9]$. At first, this was used only for AHP, but later has become applicable to general MEN problems. 
The method of MIN is the following [9]; Averaging (1.1) on $j$ for $(j, i) \in E$, we have

$$
u_{i} \fallingdotseq \sum_{j} s_{i j} u_{j} / n_{i} \quad(i=1, \cdots, n)
$$

where $n_{i}$ is the size of set $\{j \mid(j, i) \in E\}$.

The right-hand side of (2.1) is called the average of external evaluations for an object $i$, which is denoted by $\bar{u}_{i}$, and $u_{1}, \cdots, u_{n}$ making $\bar{u}_{i}$ nearest $u_{i}$ on $i(i=1, \cdots, n)$, are desirable estimates of the weights of objects $1,2, \cdots, n$. One principle for this is to decide $u_{1}, \cdots, u_{n}$ such that

$$
\min _{u_{1}, \cdots, u_{n}} \max _{i}\left\{\bar{u}_{i} / u_{i}\right\} .
$$

If the matrix $\overline{\boldsymbol{S}}\left(=\left[s_{i j} / n_{i}\right]\right)$ of this MEN problem is irreducible, the solution of $(2.2)$ is the principal eigenvector of $\overline{\boldsymbol{S}}[8,9]$. (In the field of ANP they take the principal eigenvector of $\boldsymbol{S}$ itself as the solution. If $n_{i}$ is constant on $i(i=1, \cdots, n)$, this coincides with that of $\overline{\boldsymbol{S}}$.) When $\overline{\boldsymbol{S}}$ is reducible, the solving method of MIN is not so simple, but is carried out by the general method shown in $[10,14]$.

(ii) The ALS method is another method [9] to make $\bar{u}_{i}$ near to $u_{i}(i=1, \cdots, n)$, which is to minimize the sum $D$ of squares of differences $d_{i}$ of $\bar{u}_{i}-u_{i}$,

$$
d_{i}=\bar{u}_{i}-u_{i}=\sum_{j} s_{i j} u_{j} / n_{i}-u_{i} \quad(i=1, \cdots, n) .
$$

This is a kind of least square (LS) method, but $\bar{u}_{i}$ is the averaged data, so we call this method "the averaged least square method." To minimize $D=\sum_{i} d_{i}^{2}$ we have only to take $u_{i}=0(i=1, \cdots, n)$, to have a non-sense solution. So we set the condition

$$
u_{1}+u_{2}+\cdots+u_{n}=1 \text {. }
$$

After all the ALS is the method to take $u_{1}, \cdots, u_{n}$ minimizing $D$ subject to (2.4). But we have no guarantee that the solution is positive, which is a fatal defect for our evaluation problem. The condition that the ALS method gives a positive solution is that $\overline{\boldsymbol{S}}\left(=\left[s_{i j} / n_{i}\right]\right)$ is irreducible and has the maximal eigenvalue less than 1 [9]. But there are many problems without this condition. Thus the ALS method is not well used by OR workers.

(iii) The LLS method is well known $[6,13]$. Taking the logarithm of $(1.2)$ we have

$$
\dot{u}_{i}-\dot{u}_{j} \fallingdotseq \dot{s}_{i j} \quad\left(\dot{u}_{i}=\log _{e} u_{i}, \dot{s}_{i j}=\log _{e} s_{i j}\right) .
$$

Let the error of $\dot{s}_{i j}-\left(\dot{u}_{i}-\dot{u}_{j}\right)$ be $\dot{e}_{i j}$, that is,

$$
\dot{e}_{i j}=\dot{s}_{i j}-\left(\dot{u}_{i}-\dot{u}_{j}\right)
$$

So the LLS is a method to minimize

$$
\dot{Q}=\sum_{(j, i) \in E} \dot{e}_{i j}^{2}=\sum_{(j, i) \in E}\left(\dot{s}_{i j}-\left(\dot{u}_{i}-\dot{u}_{j}\right)\right)^{2}
$$

under the condition

$$
\dot{u}_{1}+\cdots+\dot{u}_{n}=0
$$


On the evaluation problem $u_{1}, \cdots, u_{n}$ have an arbitrary (positive) constant, so we can set $u_{1} \cdot u_{2} \cdot \cdots \cdot u_{n}=1$ whose logarithm is $(2.7)$.

The LLS is a very natural method, and for an AHP with complete information, the solution of LLS is equivalent to that of the geometric mean method. This of course gives a positive solution, because exponential values obtained by the inverse transformation of logarithm are always positive.

(iv) The WLS method is also a very natural method. The principle is very simple; let

$$
e_{i j}=u_{i}-s_{i j} u_{j}, \quad(j, i) \in E
$$

and the sum $Q$ of squares of $e_{i j}$,

$$
Q=\sum_{(j, i) \in E} e_{i j}^{2}=\sum_{(j, i) \in E}\left(u_{i}-s_{i j} u_{j}\right)^{2}
$$

is minimized under the condition (2.4) on $u_{1}, \cdots, u_{n}$.

We must insist that it is proved that the solution of WLS is always positive without any conditions such as irreducibility and others like in the ALS, so we can use the WLS method without any apprehensions.

The name "WLS" (weighted LS) cannot be considered to be appropriate, because the meaning of "weight" is not so clear. But in [1] and [2] this nomenclature was already used, so we also use it.

(v) The MCS method is to minimize

$$
\chi^{2}=\sum_{(j, i) \in E}\left(s_{i j}-u_{i} / u_{j}\right)^{2} u_{j} / u_{i}
$$

on the positive $u_{1}, \cdots, u_{n}$. Formally $(2.10)$ is the same formula as $\chi^{2}$-statistics, which is the source of this name. At a glance it is difficult for us to minimize such a non-linear function as (2.10), but very simple algorithms such as the scaling method [3,4] can solve MCS problems, and further this does not need any conditions about $\boldsymbol{S}[4,15]$.

We reviewed five methods for the MEN system. There may not be any other important ones except those five methods. For example, the geometric mean method is equivalent to the LLS (for the AHP with complete information) and the direct least square method $\left(\min \sum\left(s_{i j}-u_{i} / u_{j}\right)^{2}\right)$ may have multiple solutions requiring non-linear computations to be hardly useful. And we would like to insist that the LLS and the WLS method are superior to others.

In the AHP and the ANP field, the MIN method is the most popular and may be the most frequently used. The reason may be that this is the first method proposed by the renowned T. Saaty, the pioneer in these fields. But we think that if $\boldsymbol{S}$ is reducible the analysis developed in $[10,14]$ is rather complicated and is not based on one principle throughout the series of process. As for the ALS method, it cannot give unconditionally positive solutions of weights as stated above.

On the other hand, the LLS and the WLS can be applied to any problems of MEN without any conditions except that the graph $G$ is connected, and these have the analyzing methods of errors of estimates which are described in $\S 4$ and $\S 5$. The MCS almost unconditionally gives positive solutions like the LLS and the WLS, but we cannot analyze errors of estimates by the MCS.

The LLS and the WLS have almost the same merits, and also their analyzing processes are carried out by almost the same way. The difference is only the structure of errors. That 
of the LLS is based on the logarithmic transformation of (1.2) and that of the WLS is on (1.1).

Here we solve two examples by the WLS method. The solving method of the WLS is well known and is stated in $\S 3$.

\section{Example 1.}

Consider a simple example of AHP with a comparison matrix $\boldsymbol{A}$ given by

$$
\boldsymbol{A}=\left[\begin{array}{ccc}
1 & 5 & 9 \\
1 / 5 & 1 & 3 \\
1 / 9 & 1 / 3 & 1
\end{array}\right]
$$

To solve this by the WLS we take $E=\{(2,1),(1,2),(3,1),(1,3),(3,2),(2,3)\}$. From $(3.2)$ we have

$$
\boldsymbol{X}=\left[\begin{array}{ccc}
1 & -5 & 0 \\
-1 / 5 & 1 & 0 \\
1 & 0 & -9 \\
-1 / 9 & 0 & 1 \\
0 & 1 & -3 \\
0 & -1 / 3 & 1
\end{array}\right]
$$

the normal equation (3.1) is

\begin{tabular}{cccc|c}
\hline$u_{1}$ & $u_{2}$ & $u_{3}$ & $-\lambda$ & \\
\hline 2.052346 & -5.200000 & -9.111111 & 1 & 0 \\
-5.200000 & 27.111111 & -3.333333 & 1 & 0 \\
-9.111111 & -3.333333 & 92.000000 & 1 & 0 \\
1 & 1 & 1 & 0 & 1 \\
\hline
\end{tabular}

and the solutions are $\hat{u}_{1}=0.7622, \hat{u}_{2}=0.1565, \hat{u}_{3}=0.0813, \hat{\lambda}=0.0099$. The solutions by the eigenvector method for this example are $\hat{u}_{1}=0.751, \hat{u}_{2}=0.178, \hat{u}_{3}=0.070$, which are very close to those of the WLS. It is well known that the LLS solution coincides with that of the eigenvector method, in the case with $n=3$.

Here we note that $s_{j i}$ is determined by $s_{i j}$ from (1.3), so the data in the AHP are not independent. This recognition is very important for the error analysis in $\S 5$.

\section{Example 2.}

Consider a typical ANP with the supermatrix $\boldsymbol{S}$ given by

$$
\boldsymbol{S}=\left[\begin{array}{cc}
\mathbf{0} & \boldsymbol{W} \\
\boldsymbol{U} & \mathbf{0}
\end{array}\right], \boldsymbol{U}=\left[\begin{array}{cc}
1 / 6 & 0.6 \\
1 / 3 & 0.3 \\
1 / 2 & 0.1
\end{array}\right], \boldsymbol{W}=\left[\begin{array}{ccc}
0.4 & 0.7 & 0.2 \\
0.6 & 0.3 & 0.8
\end{array}\right]
$$

For this ANP, $E=\{(3,1),(4,1),(5,1),(3,2),(4,2),(5,2),(1,3),(2,3),(1,4),(2,4),(1,5)$, $(2,5)\}$, and the matrix $\boldsymbol{X}$ in (3.2) and the normal equation in (3.1) are shown in Table 1.

The solutions are $\hat{u}_{1}=0.188906, \hat{u}_{2}=0.210889, \hat{u}_{3}=0.215728, \hat{u}_{4}=0.195837, \hat{u}_{5}=$ 0.188640 and $\hat{\lambda}=0.183522$.

On the other hand, the elements of eigenvector of the matrix $\boldsymbol{S}$ are $\hat{u}_{1}=0.175701, \hat{u}_{2}=$ $0.224299, \hat{u}_{3}=0.245794, \hat{u}_{4}=0.188785, \hat{u}_{5}=0.165421$. 
Table 1: A structure matrix and its normal equation

\begin{tabular}{cccccc}
\hline & $u_{1}$ & $u_{2}$ & $u_{3}$ & $u_{4}$ & $u_{5}$ \\
\hline$(3,1)$ & 1 & 0 & -0.4 & 0 & 0 \\
$(4,1)$ & 1 & 0 & 0 & -0.7 & 0 \\
$(5,1)$ & 1 & 0 & 0 & 0 & -0.2 \\
$(3,2)$ & 0 & 1 & -0.6 & 0 & 0 \\
$(4,2)$ & 0 & 1 & 0 & -0.3 & 0 \\
$(5,2)$ & 0 & 1 & 0 & 0 & -0.8 \\
$(1,3)$ & $-1 / 6$ & 0 & 1 & 0 & 0 \\
$(2,3)$ & 0 & -0.6 & 1 & 0 & 0 \\
$(1,4)$ & $-1 / 3$ & 0 & 0 & 1 & 0 \\
$(2,4)$ & 0 & -0.3 & 0 & 1 & 0 \\
$(1,5)$ & $-1 / 2$ & 0 & 0 & 0 & 1 \\
$(2,5)$ & 0 & -0.1 & 0 & 0 & 1 \\
\hline
\end{tabular}

\begin{tabular}{cccccc|c}
\hline$u_{1}$ & $u_{2}$ & $u_{3}$ & $u_{4}$ & $u_{5}$ & $-\lambda$ & \\
\hline 3.3889 & 0 & -0.5667 & -1.0333 & -0.7000 & 1 & 0 \\
0 & 3.4600 & -1.2000 & -0.6000 & -0.9000 & 1 & 0 \\
-0.5667 & -1.2000 & 2.5200 & 0 & 0 & 1 & 0 \\
-1.0333 & -0.6000 & 0 & 2.5800 & 0 & 1 & 0 \\
-0.7000 & -0.9000 & 0 & 0 & 2.6800 & 1 & 0 \\
1 & 1 & 1 & 1 & 1 & 0 & 1 \\
\hline
\end{tabular}

\section{The Positivity of Solutions by the WLS}

Why has the least square (LS) method, the most popular and the most powerful in the statistical field, not been used in the field of our AHP and ANP? This may be due to the eigenvector method of T. Saaty as mentioned before. Another reason is that the ALS does not always give as positive solutions.

We think that these are the origins of distrust in the LS method in our field. So the positivity of solutions by the WLS is profoundly important in our field. But the proof in [1] is valid only in the case where $\boldsymbol{X}^{T} \boldsymbol{X}$ (see (3.1)) is non-singular and its off-diagonal elements are all negative, which holds only for the AHP with complete information. Here we complete the proof for general $\boldsymbol{X}^{T} \boldsymbol{X}$.

Let the Lagrange function of WLS $\left((2.9)\right.$ and (2.4)) be $Q-2 \lambda\left(u_{1}+\cdots+u_{n}-1\right)$. Then the normal equation is given by

$$
\boldsymbol{X}^{T} \boldsymbol{X} \boldsymbol{u}=\lambda \boldsymbol{d} \quad(\text { with }(2.4))
$$

where $\boldsymbol{d}$ is the all-one column vector and the $(\nu, k)$ element $\boldsymbol{X}_{\nu k}$ of $\boldsymbol{X}$ is given by

$$
\boldsymbol{X}_{\nu i}=1, \quad \boldsymbol{X}_{\nu j}=-s_{i j} \quad \text { for } \nu=(j, i) \in E,
$$

and other elements of $\boldsymbol{X}$ are equal to zero. Note that $\nu$ denotes arc $(j, i) \in E$, and letting $r$ be the number of ares of $E$, that is, $r=|E|, \boldsymbol{X}$ is an $r \times n$ matrix. The matrix $\boldsymbol{X}$ is called the structure matrix of WLS. Of course, the solutions $\widehat{\boldsymbol{u}}=\left[\hat{u}_{1}, \cdots, \hat{u}_{n}\right]^{T}, \widehat{\lambda}$ of $(3.1)$ and (2.4) are estimates of weights of the objects. 


\section{Example 3.}

We select a simple problem of MEN defined by the graph in Figure 1. As a matter of fact, this example has peculiar significance for our research (see Example 4). The structure

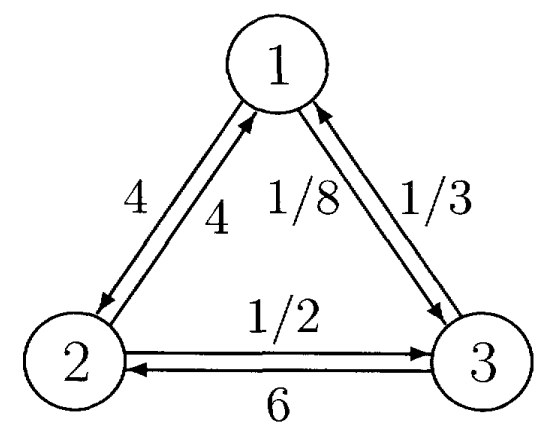

Figure 1: A graph of MEN problem

matrix $\boldsymbol{X}$ and $\boldsymbol{X}^{T} \boldsymbol{X}$ are given as follows.

$$
\begin{gathered}
\boldsymbol{X}=\left[\begin{array}{c|ccc}
\nu \backslash & 1 & 2 & (3) \\
\hline(2,1) & 1 & -4 & 0 \\
(3,1) & 1 & 0 & -1 / 3 \\
(1,2) & -4 & 1 & 0 \\
(3,2) & 0 & 1 & -6 \\
(1,3) & -1 / 8 & 0 & 1 \\
(2,3) & 0 & -1 / 2 & 1
\end{array}\right] \\
\boldsymbol{X}^{T} \boldsymbol{X}=\left[\begin{array}{cccc}
18.015625 & -8.000000 & -0.458333 \\
-8.000000 & 18.250000 & -6.500000 \\
-0.458333 & -6.500000 & 38.111111
\end{array}\right] .
\end{gathered}
$$

It is clear by the structure of WLS that $\boldsymbol{X}^{T} \boldsymbol{X}$ is non-negative definite and the offdiagonal elements are all non-positive, and if $\boldsymbol{X}^{T} \boldsymbol{X}$ is non-singular, it is positive-definite.

First we prove the positivity of Lagrange multiplier $\lambda$ in (3.1).

Lemma 1. The solution $\widehat{\lambda}$ of Lagrange multiplier in the normal equation (3.1) and (2.4) is non-negative. Moreover, we have $\widehat{\lambda}=0$ if and only if

$$
\boldsymbol{X} \boldsymbol{u}=\mathbf{0} \quad \text { or } \quad s_{i j} u_{j}=u_{i} \quad \text { for all }(j, i) \in E .
$$

Proof. From (3.1) and (2.4) we have

$$
\begin{gathered}
\boldsymbol{X}^{T} \boldsymbol{X} \boldsymbol{u}=\widehat{\lambda} \boldsymbol{d} \\
\boldsymbol{u}^{T} \boldsymbol{d}=1
\end{gathered}
$$

Multiplying (3.4) by $\boldsymbol{u}^{T}$ from the left, we get $\boldsymbol{u}^{T} \boldsymbol{X}^{T} \boldsymbol{X} \boldsymbol{u}=\widehat{\lambda} \boldsymbol{u}^{T} \boldsymbol{d}$. Then, from (3.5) we have

$$
\widehat{\lambda}=\boldsymbol{u}^{T} \boldsymbol{X}^{T} \boldsymbol{X} \boldsymbol{u}
$$

Letting $\boldsymbol{e}=\boldsymbol{X} \boldsymbol{u}$, we have $\widehat{\lambda}=\boldsymbol{e}^{T} \boldsymbol{e}$, which shows that $\widehat{\lambda} \geqq 0$. From (3.6), if $\widehat{\lambda}=0$, then $\boldsymbol{X} \boldsymbol{u}=\mathbf{0}$, and vice versa.

Lemma 2. If $\boldsymbol{X}^{T} \boldsymbol{X}$ is non-singular, then $\hat{\lambda}>0$. 
Proof. If $\widehat{\lambda}=0$, then $\boldsymbol{X}^{T} \boldsymbol{X} \boldsymbol{u}=\mathbf{0}$, so that $\boldsymbol{X}^{T} \boldsymbol{X}$ cannot be non-singular (if $\boldsymbol{X}^{T} \boldsymbol{X}$ has its inverse matrix, then $\boldsymbol{u}=\mathbf{0}$, which contradicts (2.4)). Hence, Lemma 2 follows.

Summarizing Lemma 1 and Lemma 2, we have the following theorem.

Theorem 1. If $\boldsymbol{X}^{T} \boldsymbol{X}$ is non-singular (that is, positive-definite), then $\widehat{\lambda}>0$, and if $\boldsymbol{X}^{T} \boldsymbol{X}$ is singular, then $\widehat{\lambda}=0$.

Furthermore, we have the following theorem on linear algebra.

Theorem 2. If an $n \times n$ matrix $\boldsymbol{A}=\left[a_{i j}\right]$ is positive-definite and its off-diagonal elements are all non-positive, then the solution $\boldsymbol{x}=\left[x_{1}, \cdots, x_{n}\right]^{T}$ of

$$
\boldsymbol{A x}=\boldsymbol{b}(>0)
$$

is positive $\left(x_{i}>0\right.$ for all $\left.i\right)$.

Proof. Let us solve (3.7) by the sweep-out operation. Take circled diagonal elements as pivots in Table 2 .

Table 2: Sweep-out operation $(n=3)$

\begin{tabular}{|c|ccc|c|}
\hline & $x_{1}$ & $x_{2}$ & $x_{3}$ & \\
\hline \multirow{4}{*}{ (i) } & $a_{1}$ & $a_{12}$ & $a_{13}$ & $b_{1}$ \\
& $a_{21}$ & $a_{22}$ & $a_{23}$ & $b_{2}$ \\
& $a_{31}$ & $a_{32}$ & $a_{33}$ & $b_{3}$ \\
\hline \multirow{4}{*}{ (ii) } & 1 & $a_{12}^{\prime}$ & $a_{13}^{\prime}$ & $b_{1}^{\prime}$ \\
& 0 & $a_{22}^{\prime}$ & $a_{23}^{\prime}$ & $b_{2}^{\prime}$ \\
& 0 & $a_{32}^{\prime}$ & $a_{33}^{\prime}$ & $b_{3}^{\prime}$ \\
\hline \multirow{3}{*}{ (iii) } & 1 & 0 & $a_{13}^{\prime \prime}$ & $b_{1}^{\prime \prime}$ \\
& 0 & 1 & $a_{23}^{\prime \prime}$ & $b_{2}^{\prime \prime}$ \\
& 0 & 0 & $a_{33}^{\prime \prime}$ & $b_{3}^{\prime \prime}$ \\
\hline
\end{tabular}

It is well known that these pivots $a_{11}, a_{22}^{\prime}, a_{33}^{\prime \prime}, \cdots$ are equal to the principal minors

$$
a_{11}, \quad\left|\begin{array}{ll}
a_{11} & a_{12} \\
a_{21} & a_{22}
\end{array}\right|, \quad\left|\begin{array}{lll}
a_{11} & a_{12} & a_{13} \\
a_{21} & a_{22} & a_{23} \\
a_{31} & a_{32} & a_{33}
\end{array}\right|, \cdots,
$$

respectively, and these are all positive for the positive-definite matrix $\boldsymbol{A}$.

Furthermore, the rule of sweep-out operation we have for $i \neq j$

$$
\left.a_{i j}^{\prime}=a_{i j}-\frac{a_{i 1} a_{1 j}}{a_{11}} \leqq 0 \quad \text { (because } a_{i j} \leqq 0, a_{i 1} a_{1 j} \geqq 0\right)
$$

and

$$
\left.b_{i}^{\prime}=b_{i}-\frac{a_{i 1} b_{i}}{a_{11}}>0 \quad \text { (because } b_{i}>0, a_{i 1} b_{i} \leqq 0\right) .
$$

Hence in Step (ii) in Table 2 off-diagonal elements (of the coefficient matrix) are all nonpositive and the right-hand side constant vector satisfies $\boldsymbol{b}^{\prime}>0$. The situations of Steps (ii), (iii), $\cdots$ are the same as (i). After all, we have a positive solution.

Applying Theorem 2 for our problem with $\boldsymbol{A}=\boldsymbol{X}^{T} \boldsymbol{X}$ and $\boldsymbol{b}=\lambda \boldsymbol{d}$, from Theorem 1 we have the following:

Theorem 3. If $\boldsymbol{X}^{T} \boldsymbol{X}$ is non-singular, the solutions $\widehat{\boldsymbol{u}}$ and $\widehat{\lambda}$ of $W L S$ (3.1) and (2.4) are positive, that is, $\widehat{\boldsymbol{u}}>0$ and $\hat{\lambda}>0$. 
In the case where $\boldsymbol{X}^{T} \boldsymbol{X}$ is singular, our problem results in

$$
\begin{aligned}
& \boldsymbol{X} \boldsymbol{u}=\mathbf{0}, \\
& \boldsymbol{d}^{T} \boldsymbol{u}=1,
\end{aligned}
$$

because of Theorem 1 and Lemma 1, where the rank of $\boldsymbol{X}$ is less than or equal to $n-1$.

(i) The case where the graph corresponding to rows of $\boldsymbol{X}$ is a tree $T$ : From the structure (3.2) of $\boldsymbol{X}$ it is clear that the $\operatorname{rank} \rho(\boldsymbol{X})$ of $\boldsymbol{X}$ is equal to $n-1$, and the non-zero solutions $u_{1}, \cdots, u_{n}$ of (3.8) have the same sign (see (3.3)), so that we can select $u_{1}>$ $0, \cdots, u_{n}>0$. The values of $u_{1}, \cdots, u_{n}$ are uniquely determined by (3.9).

(ii) The case where the graph $G$ corresponding to $\boldsymbol{X}$ is not a tree: Generally, the MEN graph is assumed to be connected, so the number of arcs of $G$ is greater than or equal to $n$, and we can select a tree $T$ from $G$. Since the changing of the order of rows of $\boldsymbol{X}$ has no effect on our problem, we can write

$$
\boldsymbol{X}=\left[\begin{array}{l}
\boldsymbol{X}_{\mathbf{0}} \\
\boldsymbol{X}_{\mathbf{1}}
\end{array}\right]
$$

where $\boldsymbol{X}_{\mathbf{0}}$ is the matrix composed of the rows corresponding to $T$ and $\boldsymbol{X}_{\mathbf{1}}$ is composed of the rest of the rows of $\boldsymbol{X}$. By the discussion in (i), we have the positive solution $\widehat{\boldsymbol{u}}$ of $\boldsymbol{X}_{\mathbf{0}} \boldsymbol{u}=0$, but $\widehat{\boldsymbol{u}}$ is at the same time the solution of $\boldsymbol{X}_{\mathbf{1}} \boldsymbol{u}=0$. That is because we have $\rho(\boldsymbol{X}) \leqq n-1$ and $\rho\left(\boldsymbol{X}_{\mathbf{0}}\right)=n-1$, so $\rho(\boldsymbol{X})=n-1$. Hence any row of $\boldsymbol{X}_{\mathbf{1}}$ is represented by an appropriate linear combination of rows of $\boldsymbol{X}_{\mathbf{0}}$, which shows that the solution of $\widehat{\boldsymbol{u}}$ of $\boldsymbol{X}_{\mathbf{0}} \boldsymbol{u}=0$ is the solution of $\boldsymbol{X}_{\mathbf{1}} \boldsymbol{u}=0$. Consequently, we have the positive solution of (3.8) and (3.9).

Theorem 4. Even if $\boldsymbol{X}^{T} \boldsymbol{X}$ is singular, the solution of WLS is positive. Hence the WLS always has a positive solution.

Example 4.

The normal equation (3.1) with (2.4) for Example 3 is expressed as

\begin{tabular}{cccc|c}
\hline$u_{1}$ & $u_{2}$ & $u_{3}$ & $-\lambda$ & \\
\hline 18.015625 & -8.000000 & -0.458333 & 1 & 0 \\
-8.000000 & 18.250000 & -6.500000 & 1 & 0 \\
-0.458333 & -6.500000 & 38.111111 & 1 & 0 \\
1 & 1 & 1 & 0 & 1 \\
\hline
\end{tabular}

The solutions are $\hat{u}_{1}=0.395559, \quad \hat{u}_{2}=0.431769, \hat{u}_{3}=0.172672(\widehat{\lambda}=3.592943)$. So the solutions by the WLS are all positive.

But if we apply the ALS to the data of Example 3, then we have

$$
\bar{u}_{1}=\left(4 u_{2}+u_{3} / 3\right) / 2, \bar{u}_{2}=\left(4 u_{1}+6 u_{3}\right) / 2, \bar{u}_{3}=\left(u_{1} / 8+u_{2} / 2\right) / 2 .
$$

The normal equation for this is expressed as 


\begin{tabular}{cccc|c}
\hline$u_{1}$ & $u_{2}$ & $u_{3}$ & $-\lambda$ & \\
\hline 5.003906 & -3.984375 & 5.770833 & 1 & 0 \\
-3.984375 & 5.062500 & -2.916667 & 1 & 0 \\
5.770833 & -2.916667 & 10.027778 & 1 & 0 \\
1 & 1 & 1 & 0 & 1 \\
\hline
\end{tabular}

The solutions are $\hat{u}_{1}=0.760316, \hat{u}_{2}=0.502763, \hat{u}_{3}=-0.263079$, which includes a negative component.

This example reveals the defect of the ALS, which the WLS nicely corrects.

\section{Example 5.}

Figure 2 shows an example for a singular case.

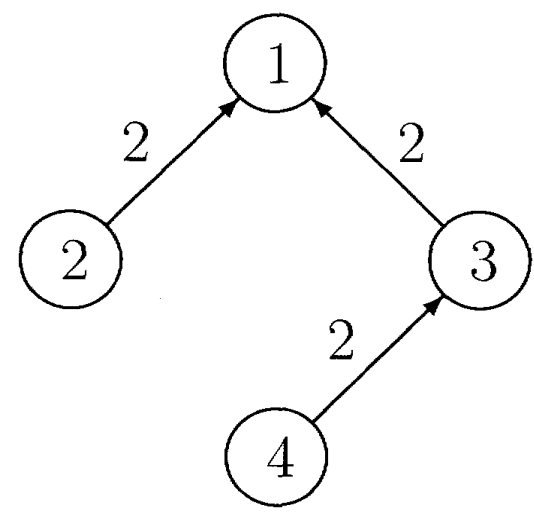

Figure 2: A singular case

We have

$$
\boldsymbol{X}=\left[\begin{array}{c|cccc} 
& u_{1} & u_{2} & u_{3} & u_{4} \\
\hline(2,1) & 1 & -2 & 0 & 0 \\
(3,1) & 1 & 0 & -2 & 0 \\
(4,3) & 0 & 0 & 1 & -2
\end{array}\right], \quad \boldsymbol{X}^{\boldsymbol{T}} \boldsymbol{X}=\left[\begin{array}{cccc}
2 & -2 & -2 & 0 \\
-2 & 4 & 0 & 0 \\
-2 & 0 & 5 & -2 \\
0 & 0 & -2 & 4
\end{array}\right]
$$

$\boldsymbol{X}^{T} \boldsymbol{X}$ is singular and the graph corresponding to $\boldsymbol{X}$ is a tree. The solutions of $\boldsymbol{X} \boldsymbol{u}=0$ are; $u_{2}=u_{1} / 2, u_{3}=u_{1} / 2, u_{4}=u_{3} / 2=u_{1} / 4$ which are determined by (3.9) as $\hat{u}_{1}=0.444$, $\hat{u}_{2}=0.222, \hat{u}_{3}=0.222, \hat{u}_{4}=0.111$.

\section{The Error Analysis on the LLS}

The error analysis on the LS with the ordinary linear model is well known (for example, see [12]). Here we extend this to the LLS (and the WLS in §5) by our specific devices. In order to analyze the errors we make the following assumptions for the errors $\dot{e}_{i j}$ in (2.6). That is, $\dot{e}_{i j}$ (for $(j, i) \in E$ ) are independent random variables such that

$$
\begin{aligned}
& E\left[\dot{e}_{i j}\right]=0 \quad \text { (the expectation of } \dot{e}_{i j} \text { is equal to zero) }, \\
& \left.V\left[\dot{e}_{i j}\right]=\sigma^{2} \quad \text { (the variance of } \dot{e}_{i j} \text { is a constant } \sigma^{2}\right) .
\end{aligned}
$$

We note that for the AHP the independence of $\dot{e}_{i j}$ (for $(j, i) \in E$ ) is not valid, because we have $\dot{e}_{i j}=-\dot{e}_{j i}$ due to (1.3). The error analysis for the AHP will be stated in "Remark" in $\S 5$. 
Let the Lagrange function of the LLS be

$$
\sum_{(j, i) \in E}\left(\dot{s}_{i j}-\left(\dot{u}_{i}-\dot{u}_{j}\right)\right)^{2}-2 \mu\left(\dot{u}_{1}+\cdots+\dot{u}_{n}\right) .
$$

Then the normal equation is given by

$$
\boldsymbol{Y}^{T} \boldsymbol{Y} \dot{\boldsymbol{u}}-\mu \boldsymbol{d}=\boldsymbol{Y}^{T} \dot{\boldsymbol{s}}
$$

with (2.7), where $\boldsymbol{Y}_{\nu k}$ is the $(\nu, k)$ element of $\boldsymbol{Y}$ with

$$
\boldsymbol{Y}_{\nu i}=1, \quad \boldsymbol{Y}_{\nu j}=-1, \quad \text { for } \nu=(j, i) \in E,
$$

and $\dot{\boldsymbol{u}}=\left[\dot{u}_{1}, \cdots, \dot{u}_{n}\right]^{T}$ and $\dot{\boldsymbol{s}}$ is the column vector whose $\nu=(j, i)$-th element is $\dot{s}_{i j}$. Then the matrix form of (2.6) becomes

$$
\dot{\boldsymbol{e}}=\dot{\boldsymbol{s}}-\boldsymbol{Y} \dot{\boldsymbol{u}}
$$

The equations (4.2) and (2.7) are equivalent to

$$
\left(\boldsymbol{Y}^{T} \boldsymbol{Y}+\boldsymbol{J}\right) \dot{\boldsymbol{u}}=\boldsymbol{Y}^{T} \dot{\boldsymbol{s}}
$$

where $\boldsymbol{J}$ is the all-one matrix. Multiplying (4.2) from the left by $\boldsymbol{d}^{T}$, we have $\boldsymbol{d}^{T} \boldsymbol{Y}^{T} \boldsymbol{Y} \dot{\boldsymbol{u}}-$ $n \mu=\boldsymbol{d}^{T} \boldsymbol{Y}^{T} \dot{\boldsymbol{s}}$, but $\boldsymbol{d}^{T} \boldsymbol{Y}^{T}=0$. Hence $\mu=0$. If $\dot{\boldsymbol{u}}$ satisfies (4.2) and (2.7), then $\dot{\boldsymbol{u}}$ also satisfies (4.5). Conversely, if $\dot{\boldsymbol{u}}$ satisfies (4.5), then multiplying (4.5) from the left by $\boldsymbol{d}^{T}$, we have $\boldsymbol{d}^{T} \boldsymbol{J} \dot{\boldsymbol{u}}=0$, which is equivalent to (2.7). Hence $\dot{\boldsymbol{u}}$ satisfies (2.7), which leads to $\boldsymbol{Y}^{T} \boldsymbol{Y} \dot{\boldsymbol{u}}=\boldsymbol{Y}^{T} \boldsymbol{s}$.

Consequently, we have only to solve one equation (4.5), where its coefficient matrix

$$
\boldsymbol{M}=\boldsymbol{Y}^{T} \boldsymbol{Y}+\boldsymbol{J}
$$

can be shown to be non-singular (see Appendix 1). Hence (4.5) has the unique solution

$$
\widehat{\dot{u}}=\boldsymbol{M}^{-1} \boldsymbol{Y}^{T} \dot{s} .
$$

Substituting this $\dot{\boldsymbol{s}}$ for $\dot{\boldsymbol{s}}$ in (4.4), we have

$$
\widehat{\hat{\boldsymbol{u}}}=\boldsymbol{M}^{-1} \boldsymbol{Y}^{T}(\boldsymbol{Y} \dot{\boldsymbol{u}}+\dot{\boldsymbol{e}})=\boldsymbol{M}^{-1} \boldsymbol{Y}^{T} \boldsymbol{Y} \dot{\boldsymbol{u}}+\boldsymbol{M}^{-1} \boldsymbol{Y}^{T} \dot{\boldsymbol{e}}
$$

But since $\boldsymbol{M}^{-1} \boldsymbol{Y}^{T} \boldsymbol{Y} \dot{\boldsymbol{u}}=\dot{\boldsymbol{u}}$ (which is equivalent to $\boldsymbol{Y}^{T} \boldsymbol{Y} \dot{\boldsymbol{u}}=\boldsymbol{M} \dot{\boldsymbol{u}}=\left(\boldsymbol{Y}^{T} \boldsymbol{Y}+\boldsymbol{J}\right) \dot{\boldsymbol{u}}=$ $\boldsymbol{Y}^{T} \boldsymbol{Y} \dot{\boldsymbol{u}}$ ), we have

$$
\widehat{\dot{u}}=\dot{\boldsymbol{u}}+\boldsymbol{M}^{-1} \boldsymbol{Y}^{T} \dot{\boldsymbol{e}}
$$

Then, the covariance matrix of $\widehat{\dot{u}}$ is given by $E\left[\boldsymbol{M}^{-1} \boldsymbol{Y}^{T} \dot{\boldsymbol{e}} \dot{\boldsymbol{e}}^{T} \boldsymbol{Y} \boldsymbol{M}^{-1}\right]$ ( $\boldsymbol{M}$ is symmetric), so that from (4.1) the variance of $\widehat{\dot{u}}_{i}$ is given by

$$
V\left[\widehat{\dot{u}}_{i}\right]=\left(\boldsymbol{M}^{-1} \boldsymbol{Y}^{T} \boldsymbol{Y} \boldsymbol{M}^{-1}\right)_{i i} \sigma^{2} \quad(i=1, \cdots, n) .
$$

Usually the error of $\widehat{\hat{u}}_{i}$ is measured by the standard error $\dot{\sigma}_{i}\left(=\sqrt{V\left[\widehat{\hat{u}}_{i}\right]}\right)$, that is, $\widehat{\hat{u}}_{i} \pm \dot{\sigma}_{i}$ is the error range of $\widehat{\dot{u}}_{i}$ (if we take the 1-sigma principle). Transforming inversely $\widehat{\dot{u}}_{i}$ and $\widehat{\vec{u}}_{i} \pm \dot{\sigma}_{i}$, we have $\widehat{u}_{i}=e^{\widehat{\hat{u}}_{i}}$. The upper limit of $\widehat{u}_{i}$ is $e^{\widehat{\dot{u}}_{i}+\dot{\sigma}_{i}}$, and the lower limit of $\widehat{u}_{i}$ is $e^{\hat{\dot{u}}_{i}-\dot{\sigma}_{i}}$. 
The formula (4.9) includes an unknown parameter $\sigma^{2}$, but we have an unbiased estimate $\widehat{\sigma^{2}}$ of $\sigma^{2}$ by the following procedure. The residue (vector) is defined by

$$
\widehat{\dot{e}}=\dot{s}-\boldsymbol{Y} \widehat{\dot{u}}
$$

which is obtained by substituting $\widehat{\dot{\boldsymbol{u}}}$ for $\dot{\boldsymbol{u}}$ in (4.4), and

$$
\widehat{\dot{Q}}=\widehat{\hat{\boldsymbol{e}}}^{T} \widehat{\dot{\boldsymbol{e}}}=\sum_{(i, j) \in E}{\widehat{\dot{\boldsymbol{e}}_{i j}}}^{2}
$$

is the sum of squares of the residues. It is well known that

$$
E[\widehat{\dot{\boldsymbol{Q}}}]=(r-(n-1)) \sigma^{2}
$$

where $r=|E|$ is the size of $E, r-(n-1)$ is often called the degree of freedom.

$$
\widehat{\sigma^{2}}=\widehat{\dot{\boldsymbol{Q}}} /(r-(n-1))
$$

is an unbiased estimate of $\sigma^{2}$. If $r-(n-1) \leqq 0$, we cannot estimate $\sigma^{2}$.

\section{The Error Analysis on the WLS}

In order to analyze the errors on the WLS we make the following assumptions for the errors $e_{i j}$ in $(2.8): e_{i j}$ (for $(j, i) \in E$ ) are independent random variables such that

$$
\begin{aligned}
& E\left[e_{i j}\right]=0 \quad\left(\text { the expectation of } e_{i j}=0\right), \\
& V\left[e_{i j}\right]=\sigma^{2} \quad\left(\text { the variance of } e_{i j} \text { is a constant } \sigma^{2}\right) .
\end{aligned}
$$

These assumptions are often taken in the statistical field.

Furthermore, the following transformation of $u_{i}$ into $v_{i}$ is useful.

$$
\boldsymbol{v}=\boldsymbol{u}-\frac{1}{n} \boldsymbol{d} \quad\left(v_{i}=u_{i}-\frac{1}{n}, \quad i=1, \cdots, n\right),
$$

which leads to the condition for $v_{1}, \cdots, v_{n}$ given as follows.

$$
\boldsymbol{d}^{T} \boldsymbol{v}=0 \quad\left(v_{1}+\cdots+v_{n}=0\right) .
$$

The matrix form of (2.8) is $\boldsymbol{e}=\boldsymbol{X} \boldsymbol{u}$, which is transformed into

$$
\begin{aligned}
\boldsymbol{e} & =\boldsymbol{X}\left(\boldsymbol{v}+\frac{1}{n} \boldsymbol{d}\right) \\
& =\boldsymbol{X} \boldsymbol{v}+\boldsymbol{x} \quad\left(\boldsymbol{x}=\frac{1}{n} \boldsymbol{X} \boldsymbol{d}\right) .
\end{aligned}
$$

And the normal equation (3.1) is transformed into

$$
\left[\begin{array}{cc}
\boldsymbol{X}^{T} \boldsymbol{X} & \boldsymbol{d} \\
\boldsymbol{d}^{T} & 0
\end{array}\right]\left[\begin{array}{c}
\boldsymbol{v} \\
-\lambda
\end{array}\right]=\left[\begin{array}{c}
-\boldsymbol{X}^{T} \boldsymbol{x} \\
0
\end{array}\right]
$$

Now if $\boldsymbol{X}^{T} \boldsymbol{X}$ has its inverse matrix $\left(\boldsymbol{X}^{T} \boldsymbol{X}\right)^{-1}$, then the inverse of coefficient matrix of $(5.5)$ is

$$
\left[\begin{array}{cc}
\boldsymbol{X}^{T} \boldsymbol{X} & \boldsymbol{d} \\
\boldsymbol{d}^{T} & 0
\end{array}\right]^{-1}=\left[\begin{array}{cc}
I+b^{-1}\left(\boldsymbol{X}^{T} \boldsymbol{X}\right)^{-1} \boldsymbol{d} \boldsymbol{d}^{T} & -\left(\boldsymbol{X}^{T} \boldsymbol{X}\right)^{-1} \boldsymbol{d} \\
-b^{-1} \boldsymbol{d}^{T} & 1
\end{array}\right]\left[\begin{array}{cc}
\left(\boldsymbol{X}^{T} \boldsymbol{X}\right)^{-1} & \mathbf{0} \\
\mathbf{0} & b^{-1}
\end{array}\right]
$$


$\left(b=-\boldsymbol{d}^{T}\left(\boldsymbol{X}^{T} \boldsymbol{X}\right)^{-1} \boldsymbol{d}\right.$ and $I$ is the unit matrix of order $\left.n\right)$.

Then the solutions $\widehat{\boldsymbol{v}}$ and $\widehat{\lambda}$ of (5.5) (that is, the WLS estimates of $\boldsymbol{v}, \lambda$ ) are given by

$$
\left[\begin{array}{c}
\widehat{\boldsymbol{v}} \\
-\widehat{\lambda}
\end{array}\right]=\left[\begin{array}{cc}
I+b^{-1}\left(\boldsymbol{X}^{T} \boldsymbol{X}\right)^{-1} \boldsymbol{d} \boldsymbol{d}^{T} & -\left(\boldsymbol{X}^{T} \boldsymbol{X}\right)^{-1} \boldsymbol{d} \\
-b^{-1} \boldsymbol{d}^{T} & 1
\end{array}\right]\left[\begin{array}{cc}
\left(\boldsymbol{X}^{T} \boldsymbol{X}\right)^{-1} & \mathbf{0} \\
\mathbf{0} & b^{-1}
\end{array}\right]\left[\begin{array}{c}
-\boldsymbol{X}^{T} \boldsymbol{x} \\
0
\end{array}\right]
$$

So we have

$$
\begin{aligned}
\widehat{\boldsymbol{v}} & =-\left(I+b^{-1}\left(\boldsymbol{X}^{T} \boldsymbol{X}\right)^{-1} \boldsymbol{d} \boldsymbol{d}^{T}\right)\left(\boldsymbol{X}^{T} \boldsymbol{X}\right)^{-1} \boldsymbol{X}^{T} \boldsymbol{x} \\
& =-\left(I+b^{-1}\left(\boldsymbol{X}^{T} \boldsymbol{X}\right)^{-1} \boldsymbol{d} \boldsymbol{d}^{T}\right)\left(\boldsymbol{X}^{T} \boldsymbol{X}\right)^{-1} \boldsymbol{X}^{T}(\boldsymbol{e}-\boldsymbol{X} \boldsymbol{v}) \quad(\text { see }(5.4)) \\
& =-\left(I+b^{-1}\left(\boldsymbol{X}^{T} \boldsymbol{X}\right)^{-1} \boldsymbol{d} \boldsymbol{d}^{T}\right)\left(\boldsymbol{X}^{T} \boldsymbol{X}\right)^{-1} \boldsymbol{X}^{T} \boldsymbol{e}+\left(I+b^{-1}\left(\boldsymbol{X}^{T} \boldsymbol{X}\right)^{-1} \boldsymbol{d} \boldsymbol{d}^{T}\right)\left(\boldsymbol{X}^{T} \boldsymbol{X}\right)^{-1} \boldsymbol{X}^{T} \boldsymbol{X} \boldsymbol{v} \\
& =\boldsymbol{v}-\left(I+b^{-1}\left(\boldsymbol{X}^{T} \boldsymbol{X}\right)^{-1} \boldsymbol{d} \boldsymbol{d}^{T}\right)\left(\boldsymbol{X}^{T} \boldsymbol{X}\right)^{-1} \boldsymbol{X}^{T} \boldsymbol{e}
\end{aligned}
$$

(because of (5.3)).

Let the coefficient matrix of $\boldsymbol{e}$ in (5.8) be $\boldsymbol{W}$, that is,

$$
\boldsymbol{W}=-\left(I+b^{-1}\left(\boldsymbol{X}^{T} \boldsymbol{X}\right)^{-1} \boldsymbol{d} \boldsymbol{d}^{T}\right)\left(\boldsymbol{X}^{T} \boldsymbol{X}\right)^{-1} \boldsymbol{X}^{T}
$$

Then the covariance matrix of $\widehat{\boldsymbol{v}}$ is $E\left[\boldsymbol{W} \boldsymbol{e} \boldsymbol{e}^{T} \boldsymbol{W}^{T}\right]$, and by (5.1) the variance of $\widehat{v_{i}}$ is

$$
V\left[\widehat{v}_{i}\right]=\left(\boldsymbol{W} \boldsymbol{W}^{T}\right)_{i i} \sigma^{2} \quad(i=1, \cdots, n)
$$

Of course, the variance of $\widehat{u_{i}}$ is the same as that of $\widehat{v_{i}}(i=1, \cdots, n)$.

We have an unbiased estimate of $\sigma^{2}$ by the following procedure.

Let $\widehat{\boldsymbol{e}}=\left[\widehat{e_{i j}}\right]$ be given by

$$
\widehat{\boldsymbol{e}}=\boldsymbol{X} \widehat{\boldsymbol{v}}+\boldsymbol{x} \quad(\operatorname{see}(5.4))
$$

whose elements $\widehat{e_{i j}}$ are often termed residues in the LS method, and

$$
\widehat{Q}=\widehat{\boldsymbol{e}}^{T} \widehat{\boldsymbol{e}}=\sum_{(i, j) \in E}{\widehat{e_{i j}}}^{2}
$$

is the sum of squares of the residues. It is well known that the expectation of $\widehat{Q}$ is given by

$$
E[\widehat{Q}]=(r-(n-1)) \sigma^{2}
$$

where $r$ is the number of paired comparisons, that is, $r=|E|$, and $r-(n-1)$ is often called the degree of freedom of error. So

$$
\widehat{\sigma^{2}}=\widehat{Q} /(r-(n-1))
$$

is an unbiased estimate of $\sigma^{2}$. If $r-(n-1) \leqq 0$, that is, the degree of freedom is zero, we cannot estimate $\sigma^{2}$. In order that the error analysis is successful, the degree of freedom must be large to some extent. 


\section{Example 6.}

The degree of freedom of Example 2 is $r-(n-1)=12-4=8, \boldsymbol{X}$ is given in Table 1 and $b=-\boldsymbol{d}^{T}\left(\boldsymbol{X}^{T} \boldsymbol{X}\right)^{-1} \boldsymbol{d}=-5.448926$.

$$
\boldsymbol{W} \boldsymbol{W}^{T}=\left[\begin{array}{rrrrr}
0.215761 & -0.092137 & -0.070320 & -0.008231 & -0.045073 \\
-0.092137 & 0.204414 & -0.007064 & -0.071104 & -0.034109 \\
-0.070320 & -0.007064 & 0.292042 & -0.113423 & -0.101235 \\
-0.008231 & -0.071104 & -0.113423 & 0.291859 & -0.099101 \\
-0.045073 & -0.034109 & -0.101235 & -0.099101 & 0.279519
\end{array}\right] .
$$

So we have

$$
\begin{aligned}
V\left[\hat{v}_{1}\right] & =\left(\boldsymbol{W} \boldsymbol{W}^{T}\right)_{11} \sigma^{2}=0.215761 \sigma^{2}, \\
V\left[\hat{v}_{2}\right] & =\left(\boldsymbol{W} \boldsymbol{W}^{T}\right)_{22} \sigma^{2}=0.204414 \sigma^{2}, \\
V\left[\hat{v}_{3}\right] & =\left(\boldsymbol{W} \boldsymbol{W}^{T}\right)_{33} \sigma^{2}=0.292042 \sigma^{2}, \\
V\left[\hat{v}_{4}\right] & =\left(\boldsymbol{W} \boldsymbol{W}^{T}\right)_{44} \sigma^{2}=0.291859 \sigma^{2}, \\
V\left[\hat{v}_{5}\right] & =\left(\boldsymbol{W} \boldsymbol{W}^{T}\right)_{55} \sigma^{2}=0.279519 \sigma^{2} .
\end{aligned}
$$

From (5.11) we have

$$
\begin{gathered}
\widehat{\boldsymbol{e}}=\boldsymbol{X} \widehat{\boldsymbol{v}}+\boldsymbol{x}=\left[\begin{array}{l}
0.102615 \\
0.051820 \\
0.151178 \\
0.081452 \\
0.152138 \\
0.059977 \\
0.184244 \\
0.089195 \\
0.132868 \\
0.132570 \\
0.094187 \\
0.167551
\end{array}\right], \\
\widehat{Q}=\sum_{(i, j) \in E}{\widehat{e_{i j}}}^{2}=0.183522, \\
\widehat{\sigma^{2}}=\widehat{Q} / 8=0.022940 .
\end{gathered}
$$

Estimating $\sigma^{2}$ in (5.15) by $\widehat{\sigma^{2}}$, we have

$$
\begin{aligned}
& V\left[\hat{u}_{1}\right]=V\left[\hat{v}_{1}\right]=0.004950, \sqrt{V\left[\hat{u}_{1}\right]}=0.070354, \\
& V\left[\hat{u}_{2}\right]=V\left[\hat{v}_{2}\right]=0.004689, \sqrt{V\left[\hat{u}_{2}\right]}=0.068479, \\
& V\left[\hat{u}_{3}\right]=V\left[\hat{v}_{3}\right]=0.006700, \sqrt{V\left[\hat{u}_{3}\right]}=0.081851, \\
& V\left[\hat{u}_{4}\right]=V\left[\hat{v}_{4}\right]=0.006695, \sqrt{V\left[\hat{u}_{4}\right]}=0.081825, \\
& V\left[\hat{u}_{5}\right]=V\left[\hat{v}_{5}\right]=0.006412, \sqrt{V\left[\hat{u}_{5}\right]}=0.080076 .
\end{aligned}
$$

The estimates $\hat{u}_{1}, \cdots, \hat{u}_{5}$ given in Example 2 have the standard errors $\sqrt{V\left[\hat{u}_{i}\right]} \quad(i=$ $1, \cdots, 5)$.

So the solutions of Example 2 have the following standard errors, 


\begin{tabular}{c|ccccc}
\hline$i$ & 1 & 2 & 3 & 4 & 5 \\
\hline$\hat{u}_{i}$ & 0.188906 & 0.210889 & 0.215728 & 0.195837 & 0.188640 \\
$\sqrt{V\left[\hat{u}_{i}\right]}$ & 0.070354 & 0.068479 & 0.081851 & 0.081825 & 0.080076 \\
\hline
\end{tabular}

Considering these errors, we have no use for the evaluations on weights of objects which are too minute.

Remark. The error analysis for the AHP.

We must pay special attention to the error analysis for the AHP. As for the LLS, we have $\dot{e}_{j i}=-\dot{e}_{i j}$, so we can delete $\dot{e}_{j i}$ in $E$. Let

$$
\bar{E}=\{(j, i) \in E \mid i<j\}, \quad \bar{r}=|\bar{E}|(=r / 2) .
$$

Then the independence and (4.1) of $\dot{e}_{i j}$ (for $(j, i) \in \bar{E}$ ) are valid. Let $\overline{\boldsymbol{Y}}$ be an $\bar{r} \times n$ submatrix of $\boldsymbol{Y}$ whose rows for $(j, i) \notin \bar{E}$ are deleted. Then we have only to use $\bar{E}, \bar{r}$ and $\overline{\boldsymbol{Y}}$ instead of $E, r$ and $\boldsymbol{Y}$, respectively.

As for the WLS, the situation is rather complex. First, from (2.8) and (1.3) we have

$$
e_{j i}=\left(u_{j}-s_{j i} u_{i}\right)=-s_{j i} e_{i j} .
$$

Hence we cannot assume the independence and the equi-variance (5.1) of $e_{i j}$. Now we have

$$
e_{i j}^{2}+e_{j i}^{2}=\left(\sqrt{1+s_{j i}^{2}} \cdot u_{i}-\sqrt{1+s_{i j}^{2}} \cdot u_{j}\right)^{2}
$$

and $Q$ of $(2.9)$ is written as

$$
Q=\sum_{(j, i) \in E} e_{i j}^{2}=\sum_{(j, i) \in \bar{E}}\left(\sqrt{1+s_{j i}^{2}} \cdot u_{i}-\sqrt{1+s_{i j}^{2}} \cdot u_{j}\right)^{2} .
$$

Let $\overline{\boldsymbol{X}}$ be an $\bar{r} \times n$ matrix with $(\nu, i)$ element of $\overline{\boldsymbol{X}}_{\nu i}$ where

$$
\overline{\boldsymbol{X}}_{\nu i}=\sqrt{1+s_{j i}^{2}}, \quad \overline{\boldsymbol{X}}_{\nu j}=-\sqrt{1+s_{i j}^{2}}, \quad \nu=(j, i) \in \bar{E} .
$$

Then the normal equation for minimizing $Q$ in (5.19) becomes

$$
\overline{\boldsymbol{X}}^{T} \overline{\boldsymbol{X}} \boldsymbol{u}=\lambda \boldsymbol{d}(\text { with (2.4)), }
$$

whose solution $\hat{\boldsymbol{u}}$ coincides with that of (3.1).

Now let

$$
\bar{e}_{i j}=\sqrt{1+s_{j i}^{2}} \cdot u_{i}-\sqrt{1+s_{i j}^{2}} \cdot u_{j}
$$

Then we can assume the independence and the equi-variance of $\bar{e}_{i j}$ (for $(j, i) \in \bar{E}$ ), that is,

$$
\begin{aligned}
& E\left[\bar{e}_{i j}\right]=0, \\
& V\left[\bar{e}_{i j}\right]=\overline{\sigma^{2}} \quad \text { for }(j, i) \in \bar{E} .
\end{aligned}
$$

Letting $\overline{\boldsymbol{e}}=\left[\bar{e}_{1}, \cdots, \bar{e}_{\bar{r}}\right]^{T}\left(e_{\nu}=e_{j, i}\right)$, we can write $(5.22)$ in a matrix form as

$$
\overline{\boldsymbol{e}}=\overline{\boldsymbol{X}} \boldsymbol{u} .
$$

We have only to use $\overline{\boldsymbol{X}}, \bar{r}$ and $\overline{\boldsymbol{e}}$ instead of $\boldsymbol{X}, r$ and $\boldsymbol{e}$, respectively, in order to have $V\left[\hat{u}_{i}\right]$ $(i=1, \cdots, n)$. 


\section{Example 7.}

The value of $\overline{\boldsymbol{X}}$ in Example 1 is given by

$$
\begin{gathered}
u_{1} \\
u_{2}
\end{gathered}
$$

The solutions of (5.21) are

$$
\hat{u}_{1}=0.7622, \hat{u}_{2}=0.1565, \hat{u}_{3}=0.0813,(\hat{\lambda}=0.0099),
$$

which of course coincide with those of Example 1. And the results of error analysis are

$$
\begin{gathered}
\widehat{Q}=0.018326(\text { by }(5.12)), \\
\widehat{\bar{\sigma}^{2}}=0.018326(\text { by }(5.13)), \\
V\left[\hat{u}_{1}\right]=0.000537, \sqrt{V\left[\hat{u}_{1}\right]}=0.023184, \\
V\left[\hat{u}_{2}\right]=0.000459, \sqrt{V\left[\hat{u}_{2}\right]}=0.021429, \\
V\left[\hat{u}_{3}\right]=0.000151, \sqrt{V\left[\hat{u}_{3}\right]}=0.012300(\text { by }(5.10)) .
\end{gathered}
$$

\section{The Analysis of the MEN Problem with Errors of Different Weights}

The simplest structure of ANP is shown in Figure 3. The set $N$ of objects is decomposed

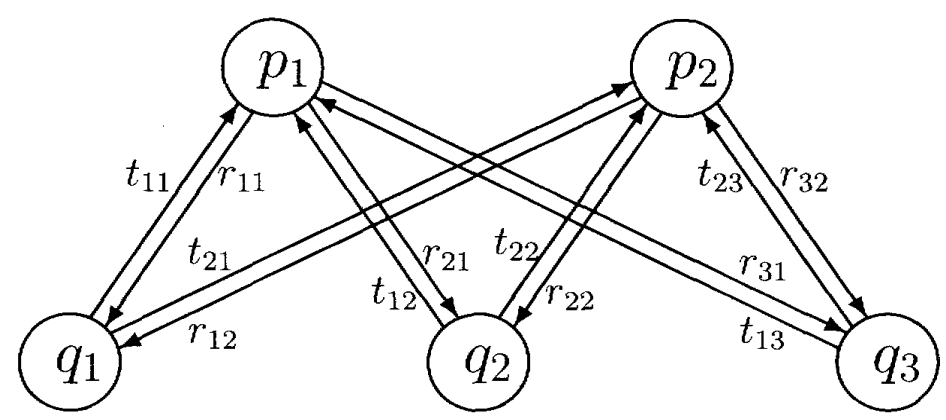

Figure 3: MECA

into two parts. The upper part $P=\left\{p_{1}, p_{2}, \cdots\right\}$ is the set of criteria and the lower part $Q=\left\{q_{1}, q_{2}, \cdots\right\}$ is the set of alternatives. Let the weight of $p_{i}$ be $u_{i}(i=1,2, \cdots)$ and that of $q_{j}$ be $v_{j}(j=1,2, \cdots)$.

Depending on this decomposition, the supermatrix $\boldsymbol{S}=\left[s_{i j}\right]$ itself is decomposed into $\boldsymbol{R}=\left[r_{i j}\right]$ and $\boldsymbol{T}=\left[t_{i j}\right]$, where $r_{i j}$ is the evaluation of alternative $q_{i} \in Q$ by criterion $p_{j} \in P$, and $t_{i j}$ is the evaluation of criterion $p_{i}$ by $q_{j}$. Then $S$ is written as

$$
S=\left[\begin{array}{ll}
0 & T \\
R & 0
\end{array}\right] .
$$

Let us call this type of ANP as the mutual evaluation of criteria and alternatives (MECA). 
As stated in [11], generally $r_{i j}$ are stable, but $t_{i j}$ are unstable, which is represented by the value of variance of errors for $r_{i j}$ and $t_{i j}$. Here after the set $\left\{(i, j) \mid q_{i} \in Q, p_{j} \in P\right\}$ $\left(\left\{(i, j) \mid p_{i} \in P, q_{j} \in Q\right\}\right)$ is denoted by $\boldsymbol{R}(\boldsymbol{T})$, which is the same symbols as the submatrix of $\boldsymbol{S}$, but we can distinguish them by the context.

Let the structure of errors be the WLS type of (2.8). Then the error of MECA are defined as

$$
\begin{array}{ll}
e_{i j}=v_{i}-r_{i j} u_{j} & \text { for }(i, j) \in \boldsymbol{R}, \\
d_{i j}=u_{i}-t_{i j} v_{j} & \text { for }(i, j) \in \boldsymbol{T}
\end{array}
$$

and the variance of $d_{i j}$ is larger than that of $e_{i j}$, that is, we assume,

$$
\begin{array}{lll}
V\left[e_{i j}\right]=\sigma^{2} & & \text { for }(i, j) \in \boldsymbol{R}, \\
V\left[d_{i j}\right]=\alpha \sigma^{2} & (\alpha>1) & \text { for }(i, j) \in \boldsymbol{T} .
\end{array}
$$

Here we treat $\alpha$ as a known parameter whose value is pre-determined by the decision maker.

Generally if the errors $e_{i j}$ have different variances $\alpha_{\nu} \sigma^{2}(\nu=(i, j))$ instead of $(5.1)$, then we can represent this by the error vector $\boldsymbol{e}$ as follows

$$
E\left[\boldsymbol{e} \boldsymbol{e}^{T}\right]=\Lambda \sigma^{2}, \quad \Lambda=\operatorname{diag}\left[\alpha_{1}, \alpha_{2}, \cdots, \alpha_{r}\right]
$$

(where $\operatorname{diag}\left[\alpha_{1}, \alpha_{2}, \cdots, \alpha_{r}\right]$ denotes the diagonal matrix whose $i$-th diagonal element is $\alpha_{i}(i=1, \cdots, r)$, and $(6.4)$ is valid because of the independence of errors.)

Multiplying $(2.8)$ or its matrix form $\boldsymbol{e}=\boldsymbol{X} \boldsymbol{u}$ by $\sqrt{\Lambda^{-1}}=\operatorname{diag}\left[\sqrt{\alpha_{1}^{-1}}, \sqrt{\alpha_{2}^{-1}}, \cdots, \sqrt{\alpha_{r}^{-1}}\right]$ from the left, we have

$$
\sqrt{\Lambda^{-1}} \boldsymbol{e}=\sqrt{\Lambda^{-1}} \boldsymbol{X} \boldsymbol{u}
$$

Then clearly the elements of $\varepsilon=\sqrt{\Lambda^{-1}} \boldsymbol{e}$ have the same variance $\sigma^{2}$. So if we use $\sqrt{\Lambda^{-1}} \boldsymbol{X}$ instead of $\boldsymbol{X}$, we can solve WLS problem with the different variances of errors. Its normal equation is

$$
\boldsymbol{X}^{T} \Lambda^{-1} \boldsymbol{X} \boldsymbol{u}=\lambda \boldsymbol{u} \quad(\text { with }(2.4))
$$

For the MECA we have

$$
\Lambda^{-1}=\operatorname{diag}\left[1, \cdots, 1, \alpha^{-1}, \cdots, \alpha^{-1}\right]
$$

where the first $|\boldsymbol{R}|$ (the next $|\boldsymbol{T}|$ ) elements of $(6.7)$ are equal to $1\left(\alpha^{-1}\right)$.

\section{Example 8.}

Consider the following data of MECA

$$
\boldsymbol{R}=\left[\begin{array}{ll}
1 / 6 & 0.6 \\
1 / 3 & 0.3 \\
1 / 2 & 0.1
\end{array}\right], \boldsymbol{T}=\left[\begin{array}{lll}
0.4 & 0.7 & 0.2 \\
0.6 & 0.3 & 0.8
\end{array}\right]
$$


The structure matrix $\boldsymbol{X}$ is

$$
\boldsymbol{X}=\left[\begin{array}{c|ccccc} 
& u_{1} & u_{2} & v_{1} & v_{2} & v_{3} \\
\hline\left(p_{1}, q_{1}\right) & -1 / 6 & 0 & 1 & 0 & 0 \\
\left(p_{1}, q_{2}\right) & -1 / 3 & 0 & 0 & 1 & 0 \\
\left(p_{1}, q_{3}\right) & -1 / 2 & 0 & 0 & 0 & 1 \\
\left(p_{2}, q_{1}\right) & 0 & -0.6 & 1 & 0 & 0 \\
\left(p_{2}, q_{2}\right) & 0 & -0.3 & 0 & 1 & 0 \\
\left(p_{2}, q_{3}\right) & 0 & -0.1 & 0 & 0 & 1 \\
\left(q_{1}, p_{1}\right) & 1 & 0 & -0.4 & 0 & 0 \\
\left(q_{1}, p_{2}\right) & 0 & 1 & -0.6 & 0 & 0 \\
\left(q_{2}, p_{1}\right) & 1 & 0 & 0 & -0.7 & 0 \\
\left(q_{2}, p_{2}\right) & 0 & 1 & 0 & -0.3 & 0 \\
\left(q_{3}, p_{1}\right) & 1 & 0 & 0 & 0 & -0.2 \\
\left(q_{3}, p_{2}\right) & 0 & 1 & 0 & 0 & -0.8
\end{array}\right]
$$

Selecting $\alpha=2$, we have its normal equation by (6.6),

\begin{tabular}{cccccc|c}
\hline$u_{1}$ & $u_{2}$ & $v_{1}$ & $v_{2}$ & $v_{3}$ & $-\lambda$ & \\
\hline 1.888889 & 0 & -0.366667 & -0.683333 & -0.600000 & 1 & 0 \\
0 & 1.960000 & -0.900000 & -0.450000 & -0.500000 & 1 & 0 \\
-0.366667 & -0.900000 & 2.260000 & 0 & 0 & 1 & 0 \\
-0.683333 & -0.450000 & 0 & 2.290000 & 0 & 1 & 0 \\
-0.600000 & -0.500000 & 0 & 0 & 2.340000 & 1 & 0 \\
1 & 1 & 1 & 1 & 1 & 0 & 1 \\
\hline
\end{tabular}

The solutions are

$$
\hat{u}_{1}=0.225540, \hat{u}_{2}=0.240508, \hat{v}_{1}=0.192585, \hat{v}_{2}=0.173989, \hat{v}_{3}=0.167378
$$

This problem is the same as Example 2, which is solved on the assumption of constant variance (5.1) and the results are

$$
\hat{u}_{1}=0.188906, \hat{u}_{2}=0.210889, \hat{v}_{1}=0.215728, \hat{v}_{2}=0.195837, \hat{v}_{3}=0.188640 .
$$

These results $\left(\hat{u}_{1}\right.$ and $\hat{u}_{2}$ in (6.9) are higher than those in $(6.10)$ ) are interpreted by the following claim. If we have the assumption that evaluation from criteria $(C)$ to alternatives (A) has higher accuracy than that from $\mathrm{A}$ to $\mathrm{C}$, the estimation $\left(\hat{u}_{1}\right.$ and $\left.\hat{u}_{2}\right)$ of weights of $\mathrm{C}$ becomes higher than that without such an assumption.

We have the variances of estimates $\hat{u}_{i}$ for the general problem by only using $\sqrt{\Lambda^{-1}} \boldsymbol{X}$ instead of $\boldsymbol{X}$ in (5.9) and (5.10), that is, $V\left[\hat{u}_{i}\right]=\left(\boldsymbol{U} \boldsymbol{U}^{T}\right)_{i i} \rho^{2}(i=1, \cdots, n)$,

$$
\begin{gathered}
\boldsymbol{U}=\left(I+\beta^{-1}\left(\boldsymbol{X}^{T} \Lambda^{-1} \boldsymbol{X}\right)^{-1} \boldsymbol{d} \boldsymbol{d}^{T}\right)\left(\boldsymbol{X}^{T} \Lambda^{-1} \boldsymbol{X}\right)^{-1} \boldsymbol{X}^{T} \sqrt{\Lambda^{-1}}, \\
\beta=-\boldsymbol{d}^{T}\left(\boldsymbol{X}^{T} \Lambda^{-1} \boldsymbol{X}\right)^{-1} \boldsymbol{d}, \\
\widehat{\rho^{2}}=\widehat{\boldsymbol{\varepsilon}}^{T} \widehat{\varepsilon} /(r-(n-1)), \\
\widehat{\varepsilon}=\sqrt{\Lambda^{-1}} \boldsymbol{X} \widehat{\boldsymbol{u}} .
\end{gathered}
$$




\section{Example 9.}

The estimates $\hat{u}_{1}, \hat{u}_{2}, \hat{v}_{1}, \hat{v}_{2}, \hat{v}_{3}$ in (6.9) have the variances

$$
\begin{aligned}
& V\left[\hat{u}_{1}\right]=\left(\boldsymbol{U} \boldsymbol{U}^{T}\right)_{11} \rho^{2}=0.365310 \rho^{2}, \\
& V\left[\hat{u}_{2}\right]=\left(\boldsymbol{U} \boldsymbol{U}^{T}\right)_{22} \rho^{2}=0.348378 \rho^{2}, \\
& V\left[\hat{v}_{1}\right]=\left(\boldsymbol{U} \boldsymbol{U}^{T}\right)_{33} \rho^{2}=0.339792 \rho^{2}, \\
& V\left[\hat{v}_{2}\right]=\left(\boldsymbol{U} \boldsymbol{U}^{T}\right)_{44} \rho^{2}=0.335089 \rho^{2}, \\
& V\left[\hat{v}_{3}\right]=\left(\boldsymbol{U} \boldsymbol{U}^{T}\right)_{55} \rho^{2}=0.328917 \rho^{2} .
\end{aligned}
$$

where $\rho^{2}$ is estimated by $\widehat{\rho^{2}}(6.12)$.

$$
\begin{aligned}
\widehat{\rho^{2}} & =\widehat{\boldsymbol{u}}^{T} \boldsymbol{X}^{T} \Lambda^{-1} \boldsymbol{X} \widehat{\boldsymbol{u}} /(12-4) \quad(\boldsymbol{X} \text { is in }(6.8)) \\
& =0.017011
\end{aligned}
$$

Table 3 is the comparison of estimates and its standard errors of solutions on the constant-variance and the different-variance assumption;

Table 3: The comparison of solutions on constant and different variance

\begin{tabular}{c|c|cc|c|ccc}
\hline & $i$ & 1 & 2 & $i$ & 1 & 2 & 3 \\
\hline constant & $\hat{u}_{i}$ & 0.188906 & 0.210889 & $\hat{v}_{i}$ & 0.215728 & 0.195837 & 0.188640 \\
variance & $\sqrt{V\left[\hat{u}_{i}\right]}$ & 0.070354 & 0.068479 & $\sqrt{V\left[\hat{v}_{i}\right]}$ & 0.081851 & 0.081825 & 0.080076 \\
\hline different & $\hat{u}_{i}$ & 0.225540 & 0.240508 & $\hat{v}_{i}$ & 0.192585 & 0.173989 & 0.167378 \\
variance & $\sqrt{V\left[\hat{u}_{i}\right]}$ & 0.078830 & 0.076982 & $\sqrt{V\left[\hat{v}_{i}\right]}$ & 0.076027 & 0.075499 & 0.074801 \\
\hline
\end{tabular}

\section{The Solution Method of a Reducible ANP by the WLS and the LLS}

In [14] there is an ANP problem, whose supermatrix is

$$
\boldsymbol{S}=\left[\begin{array}{c|cccccc} 
& (1) & (2) & (3) & (4) & (5) & 6 \\
\hline(1) & 0 & 0 & 0 & 0 & 0 & 0 \\
(2) & x & 0 & 0 & 1 / 4 & 3 & 2 \\
(3) & y & 0 & 0 & 1 & 1 & 1 \\
(4) & 0 & 1 & 1 & 0 & 0 & 0 \\
(5) & 0 & 2 & 1 / 2 & 0 & 0 & 0 \\
(6) & 0 & 3 & 1 / 6 & 0 & 0 & 0
\end{array}\right]
$$

and its graph $G$ is shown in Figure 4. ( $\boldsymbol{S}$ is not stochastic, so that it may be correct for us to call this a MEN rather than an ANP.) Note that $x$ and $y$ in $\boldsymbol{S}$ are treated as parameters in [14].

$\boldsymbol{S}$ is a reducible matrix, so we cannot solve this by the simple eigenvector method. In [14] this is solved by the general solution method for the ANP, which we think, however, is a rather complex method. Here we would like to solve this problem by the WLS and the LLS. 


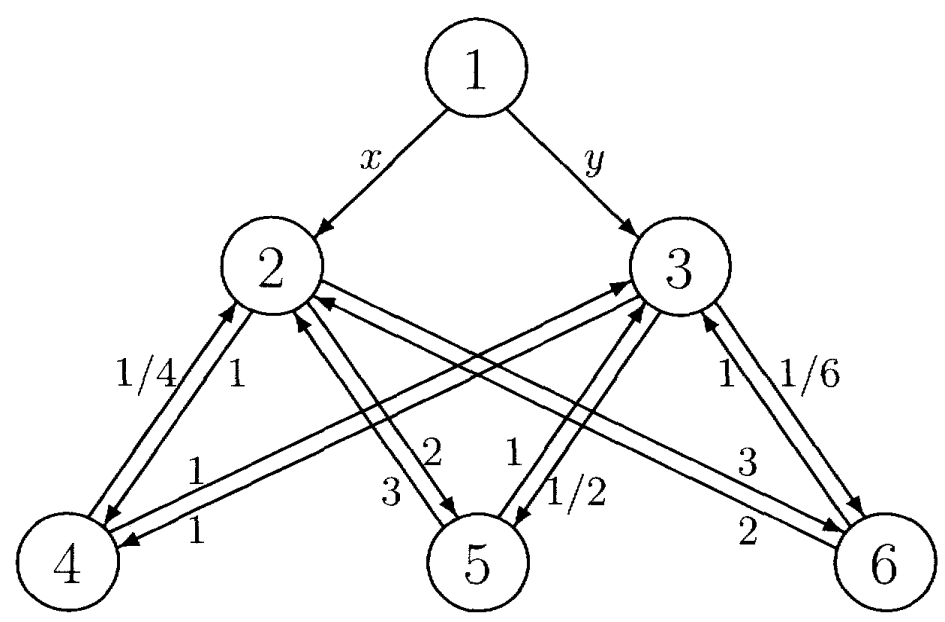

Figure 4: A reducible ANP

\subsection{The solution method by the WLS}

The structure matrix $\boldsymbol{X}$ for this problem is

$$
\boldsymbol{X}=\left[\begin{array}{c|cccccc} 
& u_{1} & u_{2} & u_{3} & u_{4} & u_{5} & u_{6} \\
\hline(1,2) & -x & 1 & & & & \\
(4,2) & & 1 & & -1 / 4 & & \\
(5,2) & & 1 & & & -3 & \\
(6,2) & & 1 & & & & -2 \\
(1,3) & -y & & 1 & & & \\
(4,3) & & & 1 & -1 & & \\
(5,3) & & & 1 & & -1 & \\
(6,3) & & & 1 & & & -1 \\
(2,4) & & -1 & & 1 & & \\
(3,4) & & & -1 & 1 & & \\
(2,5) & & -2 & & & 1 & \\
(3,5) & & & -1 / 2 & & 1 & \\
(2,6) & & -3 & & & & 1 \\
(3,6) & & & -1 / 6 & & & 1
\end{array}\right] .
$$

(Blank elements are zeros)

For $x=y=0.5$, the normal equation is

\begin{tabular}{ccccccc|c}
\hline$u_{1}$ & $u_{2}$ & $u_{3}$ & $u_{4}$ & $u_{5}$ & $u_{6}$ & $-\lambda$ & \\
\hline 0.500000 & -0.500000 & -0.500000 & 0 & 0 & 0 & 1 & 0 \\
-0.500000 & 18.000000 & 0 & -1.250000 & -5.000000 & -5.000000 & 1 & 0 \\
-0.500000 & 0 & 5.277778 & -2.000000 & -1.500000 & -1.166667 & 1 & 0 \\
0 & -1.250000 & -2.000000 & 3.062500 & 0 & 0 & 1 & 0 \\
0 & -5.000000 & -1.500000 & 0 & 12.000000 & 0 & 1 & 0 \\
0 & -5.000000 & -1.166667 & 0 & 0 & 7.000000 & 1 & 0 \\
1 & 1 & 1 & 1 & 1 & 1 & 0 & 1 \\
\hline
\end{tabular}

and the solutions are $\hat{u}_{1}=0.442035, \hat{u}_{2}=0.072213, \hat{u}_{3}=0.163276, \hat{u}_{4}=0.169826, \hat{u}_{5}=$ $0.059104, \hat{u}_{6}=0.093546(\widehat{\lambda}=0.103273)$. 
Here we compare these results with those by the generalized ANP [14]. Neglecting $\hat{u}_{1}$ and standardizing $\hat{u}_{2}, \cdots, \hat{u}_{6}$, we have

\begin{tabular}{cccccc}
\hline$\hat{u}_{2}$ & $\hat{u}_{3}$ & $\hat{u}_{4}$ & $\hat{u}_{5}$ & $\hat{u}_{6}$ & \\
\hline .129 & .293 & .304 & .106 & .168 & (WLS) \\
.338 & .312 & .089 & .114 & .146 & $([14])$ \\
\hline
\end{tabular}

We recognize two kinds of differences between the WLS and the method by [14].

The first: the values of $\hat{u}_{2}$ and $\hat{u}_{3}$ (in the second stratum of Figure 4) by the WLS are lower than those by [14].

The second: $\hat{u}_{2}<\hat{u}_{3}$ by the WLS but $\hat{u}_{2} \fallingdotseq \hat{u}_{3}$ by [14].

The method in [14] is based on the MIN whose principle (2.1) makes $\hat{u}_{i}$ to be near to the average of evaluations from only other objects. But the WLS considers the evaluations of object $i$ to other objects, too. So if an object $i$ gives higher evaluations to inferior objects, this makes $\hat{u}_{i}$ to be lower, which [14] does not consider.

Consider the evaluations of objects 2 and 3 from other objects in this example which are given by

$$
\hat{u}_{2} \fallingdotseq \frac{1}{4} \hat{u}_{4}, \hat{u}_{2} \fallingdotseq 3 \hat{u}_{5}, \hat{u}_{2} \fallingdotseq 2 \hat{u}_{6}, \quad \text { and } \quad \hat{u}_{3} \fallingdotseq \hat{u}_{4}, \hat{u}_{3} \fallingdotseq \hat{u}_{5}, \hat{u}_{3} \fallingdotseq \hat{u}_{6} \text {. }
$$

This shows object 2 has rather higher evaluation from others than object 3 . But evaluations of others by these two objects are

$$
\hat{u}_{4} \fallingdotseq \hat{u}_{2}, \hat{u}_{5} \fallingdotseq \hat{2} u_{2}, \hat{u}_{6} \fallingdotseq \hat{3} u_{2}, \quad \text { and } \quad \hat{u}_{4} \fallingdotseq \hat{u}_{3}, \hat{u}_{5} \fallingdotseq \frac{1}{2} \hat{u}_{3}, \hat{u}_{6} \fallingdotseq \frac{1}{6} \hat{u}_{3} \text {. }
$$

This shows object 2 gives higher evaluation to others than object 3 . This causes the second fact, as mentioned above.

The reason of the first fact is that objects 2 and 3 have the evaluations from object 1 , but objects 4,5 and 6 have not.

By (5.10) the variances of $\hat{u}_{i}(i=1, \cdots, 6)$ are

$$
\begin{aligned}
& V\left[\hat{u}_{1}\right]=0.839007 \sigma^{2}, V\left[\hat{u}_{2}\right]=0.062586 \sigma^{2}, V\left[\hat{u}_{3}\right]=0.156707 \sigma^{2}, \\
& V\left[\hat{u}_{4}\right]=0.297168 \sigma^{2}, V\left[\hat{u}_{5}\right]=0.086199 \sigma^{2}, V\left[\hat{u}_{6}\right]=0.151517 \sigma^{2} .
\end{aligned}
$$

By $(5.14) \widehat{\sigma^{2}}$ is given by

$$
\widehat{\sigma^{2}}=0.011475
$$

So the standard errors are

$\sqrt{V\left[\hat{u}_{1}\right]}=0.098119, \sqrt{V\left[\hat{u}_{2}\right]}=0.026799, \sqrt{V\left[\hat{u}_{3}\right]}=0.042405, \sqrt{V\left[\hat{u}_{4}\right]}=0.058395, \sqrt{V\left[\hat{u}_{5}\right]}$ $=0.031450, \sqrt{V\left[\hat{u}_{6}\right]}=0.041697$.

\subsection{The solution method by the LLS}

As mentioned in $\S 2$, although the LLS is a natural method, this is not used so often in the fields of the AHP and the ANP. The LLS can solve even reducible ANP problems by almost the same way as the WLS. 
Now we solve the ANP with $S$ in (7.1) and the graph $G$ in Figure 4. From (2.5) and (4.3) we have the structure matrix $\boldsymbol{Y}$ and $\dot{\boldsymbol{s}}$ as follows.

\begin{tabular}{l|llllll|l}
\hline & \multicolumn{5}{|c}{$\boldsymbol{Y}$} & \multicolumn{1}{c}{$\dot{\boldsymbol{s}}$} \\
& $\dot{u}_{1}$ & $\dot{u}_{2}$ & $\dot{u}_{3}$ & $\dot{u}_{4}$ & $\dot{u}_{5}$ & $\dot{u}_{6}$ & \\
\hline$(1,2)$ & -1 & 1 & & & & & $\log x$ \\
$(4,2)$ & & 1 & & -1 & & & $-\log 4=-1.386294$ \\
$(5,2)$ & & 1 & & & -1 & & $\log 3=1.098612$ \\
$(6,2)$ & & 1 & & & & -1 & $\log 2=0.693147$ \\
$(1,3)$ & -1 & & 1 & & & & $\log y$ \\
$(4,3)$ & & & 1 & -1 & & & $\log 1=0$ \\
$(5,3)$ & & & 1 & & -1 & & $\log 1=0$ \\
$(6,3)$ & & & 1 & & & -1 & $\log 1=0$ \\
$(2,4)$ & & -1 & & 1 & & & $\log 1=0$ \\
$(3,4)$ & & & -1 & 1 & & & $\log 1=0$ \\
$(2,5)$ & & -1 & & & 1 & & $\log 2=0.693147$ \\
$(3,5)$ & & & -1 & & 1 & & $-\log 2=-0.693147$ \\
$(2,6)$ & & -1 & & & & 1 & $\log 3=1.098612$ \\
$(3,6)$ & & & -1 & & & 1 & $-\log 6=-1.791759$ \\
\hline
\end{tabular}

(Blank elements are zeros and the base of $\log$ is $e$ )

For $x=y=0.5$, the normal equation (4.2) is

\begin{tabular}{rrrrrrr|r}
\hline$\dot{u}_{1}$ & $\dot{u}_{2}$ & $\dot{u}_{3}$ & $\dot{u}_{4}$ & $\dot{u}_{5}$ & $\dot{u}_{6}$ & $-\mu$ & \\
\hline 2 & -1 & -1 & 0 & 0 & 0 & 1 & 1.386294 \\
-1 & 7 & 0 & -2 & -2 & -2 & 1 & -2.079442 \\
-1 & 0 & 7 & -2 & -2 & -2 & 1 & 1.791759 \\
0 & -2 & -2 & 4 & 0 & 0 & 1 & 1.386294 \\
0 & -2 & -2 & 0 & 4 & 0 & 1 & -1.098612 \\
0 & -2 & -2 & 0 & 0 & 4 & 1 & -1.386294 \\
1 & 1 & 1 & 1 & 1 & 1 & 0 & 0 \\
\hline
\end{tabular}

and the solutions are $\widehat{\dot{u}}_{1}=0.623398, \widehat{\dot{u}}_{2}=-0.346263, \widehat{\dot{u}}_{3}=0.206765, \widehat{\dot{u}}_{4}=0.276825$, $\widehat{\vec{u}}_{5}=-0.344402, \widehat{\dot{u}}_{6}=-0.416323$, and $\hat{u}_{1}=0.287435, \hat{u}_{2}=0.108998, \hat{u}_{3}=0.189495$, $\hat{u}_{4}=0.203247, \hat{u}_{5}=0.109202, \hat{u}_{6}=0.101623$. (where $\hat{u}_{i}$ is standardized by $\sum_{i} \hat{u}_{i}=1$ ).

By (4.9) the variances of $\widehat{\dot{u}}_{i}(i=1, \cdots, 6)$ are

$$
\begin{aligned}
& V\left[\widehat{\dot{u}}_{1}\right]=0.368056 \sigma^{2}, V\left[\widehat{\dot{u}}_{2}\right]=0.106151 \sigma^{2}, V\left[\widehat{\dot{u}}_{3}\right]=0.106151 \sigma^{2}, \\
& V\left[\widehat{\dot{u}}_{4}\right]=0.201389 \sigma^{2}, V\left[\widehat{\dot{u}}_{5}\right]=0.201389 \sigma^{2}, V\left[\widehat{\dot{u}}_{6}\right]=0.201389 \sigma^{2},
\end{aligned}
$$

and $\sigma^{2}$ is estimated by (4.11) and (4.12) as

$$
\widehat{\sigma^{2}}=0.739376
$$

So the standard error, $\dot{\sigma}_{i}=\sqrt{V\left[\hat{\vec{u}}_{i}\right]}(i=1, \cdots, 6)$, are $\dot{\sigma}_{1}=0.521662, \dot{\sigma}_{2}=0.280152$, $\dot{\sigma}_{3}=0.280152, \dot{\sigma}_{4}=0.385878, \dot{\sigma}_{5}=0.385878, \dot{\sigma}_{6}=0.385878$. Therefore the upper (lower) 
limits of $\hat{u}_{i}, e^{\widehat{\vec{u}}_{i}+\dot{\sigma}_{i}}\left(e^{\widehat{\dot{u}}_{i}-\dot{\sigma}_{i}}\right)$, are given by

\begin{tabular}{lrr}
\hline & the upper limit & the lower limit \\
\hline$\hat{u}_{1}$ & 3.142631 & 1.107091 \\
$\hat{u}_{2}$ & 0.936027 & 0.534504 \\
$\hat{u}_{3}$ & 1.627293 & 0.929241 \\
$\hat{u}_{4}$ & 1.940029 & 0.896682 \\
$\hat{u}_{5}$ & 1.042348 & 0.481774 \\
$\hat{u}_{6}$ & 0.970015 & 0.448341 \\
\hline
\end{tabular}

\section{Conclusion}

This paper insists that the WLS and the LLS are superior to other methods (the MIN, the ALS and the MCS) to solve MEN problems ( $(2)$. Both methods can solve MEN problems without any restrictions about the matrix $\boldsymbol{S}$. (The MCS has this property, but it does not have the error analyzing methods.) This is based on mainly that the WLS always gives positive solutions, which is proved in $\S 3$. (The proof in [1] is restricted to the case where $\boldsymbol{X}^{T} \boldsymbol{X}$ is non-singular.) Another reason is that the WLS and the LLS have the error analyzing methods which are stated in $\S 4$ and $\S 5$. Furthermore, the WLS and the LLS can solve MEN problems with the different variances of errors ( $(6)$. In $\S 7$, the ANP problem with the reducible supermatrix is solved by the WLS and the LLS, and is compared with the generalized ANP method [14].

\section{A. Appendix 1}

Here we prove

$$
\boldsymbol{M}=\boldsymbol{Y}^{T} \boldsymbol{Y}+\boldsymbol{J} \quad(\text { for } \boldsymbol{Y} \text { in }(4.3))
$$

is non-singular. Firstly we write

$$
\boldsymbol{M}=\boldsymbol{Y}^{T} \boldsymbol{Y}+\boldsymbol{J}=\left[\begin{array}{ll}
\boldsymbol{Y}^{T} & \boldsymbol{d}
\end{array}\right]\left[\begin{array}{c}
\boldsymbol{Y} \\
\boldsymbol{d}^{T}
\end{array}\right]
$$

So in order to prove that $\boldsymbol{M}$ is non-singular, $\left[\begin{array}{c}\boldsymbol{Y} \\ \boldsymbol{d}^{T}\end{array}\right]$ is required to have the rank equal to $n$, which is equivalent to that the solution $\boldsymbol{x}$ of

$$
\left[\begin{array}{c}
\boldsymbol{Y} \\
\boldsymbol{d}^{T}
\end{array}\right] \boldsymbol{x}=\mathbf{0}
$$

is a zero vector. Note that the number of rows of $\left[\begin{array}{c}\boldsymbol{Y} \\ \boldsymbol{d}^{T}\end{array}\right]$ is $r+1(r=|\boldsymbol{E}|)$.

The structure of $\boldsymbol{Y}$ in (A.3) is diverse, but the least value of $r$ is equal to $n-1$ (this is the least number of graph $G$ of this MEN to be connected), when $G$ becomes a tree. So if the solution of (A.3) is shown to be zero for $\boldsymbol{Y}$ whose graph is an arbitrary tree, then it is valid for $\boldsymbol{Y}$ with any graph.

When the graph corresponding to $\boldsymbol{Y}$ is a tree, any two elements of solution $\boldsymbol{x}=$ $\left[x_{1}, \cdots, x_{n}\right]^{T}$ are the same, that is, $x_{1}=\cdots=x_{n}$ from the structure (4.3) of $\boldsymbol{Y}$. So $\boldsymbol{d}^{T} \boldsymbol{x}=0$ requires $\boldsymbol{x}=\mathbf{0}$. 


\section{Acknowledgements}

The authors wish to express our heart-felt gratitude for intrinsic and useful comments from Professor Kazuyuki Sekitani of Shizuoka University, and heartily thank one of our anonymous reviewers who pointed out our intrinsic misunderstanding.

\section{References}

[1] E. Blankmeyer: Approaches to consistency adjustment. Journal of Optimization Theory and Applications, 54 (1987), 479-488.

[2] A.T.W. Chu, R.E. Kalaba and K. Spingarn: A comparison of two methods for determining the weights of belonging to fuzzy sets. Journal of Optimization Theory and Applications, 27 (1979), 531-538.

[3] K. Genma, Y. Kato and K. Sekitani: Matrix balancing problem and binary AHP. Journal of the Operations Research Society of Japan, 50 (2007), 515-539.

[4] R.E. Jensen: Comparisons of eigenvector, least squares, chi square and logarithmic least square methods of scaling a reciprocal matrix. Trinity University, USA, Working Paper, 127 (1984).

[5] T.L. Saaty: The Analytic Hierarchy Process (McGraw-Hill, New York, 1980).

[6] T.L. Saaty and L.G. Vargas: Comparison of eigenvalues, logarithmic least squares, and least square methods in estimating ratios. Mathematical Modeling, 5 (1984), 309-324.

[7] T.L. Saaty: The Analytic Network Process (RWS Publications, Pittsburgh, 1996).

[8] K. Sekitani and N. Yamaki: A logical interpretation for eigenvalue method in AHP. Journal of the Operations Research Society of Japan, 42 (1999), 219-232.

[9] K. Sekitani: Chapter 7 in AHP no Riron to Jissai. In: E. Kinoshita (ed.), AHP no Riron to Jissai (Nikkagiren, Tokyo, 2000) (In Japanese).

[10] K. Sekitani and I. Takahashi: A unified model and analysis for AHP and ANP. Journal of the Operations Research Society of Japan, 44 (2001), 67-89.

[11] K. Sekitani and I. Takahashi: A new approach of revising unstable data in ANP by Bayes theorem. Journal of the Operations Research Society of Japan, 48 (2005), 24-40.

[12] I. Takahashi, R. Kobayashi and Y. Koyanagi: Tōkei kaiseki (Baifūkan, Tokyo, 1992) (In Japanese).

[13] I. Takahashi: AHP kara ANP eno shomonndai I VI. Communications of the Operations Research Society of Japan, 43 (1998) (In Japanese).

[14] I. Takahashi: Chapter 2 in AHP no Riron to Jissai. In: E. Kinoshita (ed.), AHP no Riron to Jissai (Nikkagiren, Tokyo, 2000) (In Japanese).

[15] Q.H. Vuong and W. Wang: Minimum chi-square estimation and tests for model selection. Journal of Econometrics, 56 (1993), 141-168.

Kazutomo Nishizawa

Department of Mathematical Information Engineering, College of Industrial Technology, Nihon University 1-2-1 Izumicho, Narashino, Chiba 275-8575, Japan

E-mail: nishizawa.kazutomo@nihon-u.ac.jp 